\title{
Sorption of Pyrene by Clay Minerals Coated with Dissolved Organic Matter (DOM) from Landfill Leachate
}

\author{
Pingxiao Wu, ${ }^{1}$ Yong Wen, ${ }^{2,3}$ Yun Xiang, ${ }^{2,3}$ Jianbing Zhou, \\ Jianwei $\mathrm{Du}^{2,3}$ Nengwu Zhu, ${ }^{1}$ and Ping $\mathrm{Li}^{1}$ \\ ${ }^{1}$ College of Environment and Energy, South China University of Technology, Guangzhou 510006, China \\ ${ }^{2}$ South China Institute of Environmental Sciences of the Ministry of Environmental Protection, Guangzhou 510655, China \\ ${ }^{3}$ The Key Laboratory of Water and Air Pollution Control of Guangdong Province, Guangzhou 510655, China \\ Correspondence should be addressed to Yong Wen; wenyong@scies.org
}

Received 4 July 2014; Accepted 12 August 2014

Academic Editor: Jian Lu

Copyright (C) 2015 Pingxiao Wu et al. This is an open access article distributed under the Creative Commons Attribution License, which permits unrestricted use, distribution, and reproduction in any medium, provided the original work is properly cited.

\begin{abstract}
Interactions of dissolved organic matter (DOM) from landfill leachate with clays could affect significantly the fate of hydrophobic organic compounds (HOCs) in soils. The complexes of montmorillonite (MT) and kaolinite (KL) with DOM extracted from landfill leachate were prepared under controlled conditions, termed CMT and CKL, respectively. The bare clays and their complexes were characterized by powder X-ray diffraction (XRD), Fourier transform infrared (FTIR), thermogravimetry (TG), differential scanning calorimetry (DSC), and scanning electron microscopy (SEM). Batch experiments were designed to investigate the sorption behavior of pyrene onto the clays in the presence of DOM. The maximum sorption capacities of pyrene on MT, CMT, KL, and CKL were $22.18,38.96,42.00$, and $44.99 \mu \mathrm{g} \cdot \mathrm{g}^{-1}$, respectively, at the initial concentration of $1000 \pm 150 \mu \mathrm{g} \cdot \mathrm{L}^{-1}$. The sorption isotherms of pyrene by the bare clays followed the Henry model well, whereas the Freundlich sorption isotherm provided a better fit to the equilibrium data of the sorption by the complexes. The role of montmorillonite and kaolinite complexes with DOM in the retention of pyrene in soils was different. This may be due to the different crystal structures between montmorillonite and kaolinite.
\end{abstract}

\section{Introduction}

The contamination of soil and groundwater attributed to the migration of municipal solid waste (MSW) leachate from dilute-and-disperse landfills has aroused much concern [1-3]. High content of dissolved organic matter (DOM) is present in the landfill leachate, which significantly affects the sorption and transport of various pollutants in soils [4-7], and adsorption of DOM onto clay minerals in soils is an important process in the natural environment. After soils were contaminated by landfill leachate, certain functional groups of DOM could form complex with clay minerals owing to the fine particle size, favorable microspores, and high sorption capacity $[8,9]$. Thus, interactions of DOM from landfill leachate with clays could affect significantly the fate and reactivity of organic pollutants in soils [9, 10], such as HOCs. Besides, one of the traditional liners is geosynthetic clay liners (GCLs), consisting primarily of nature clays or processed clays [11]. Thus, clay minerals also play an important role in the persistence of organic pollutants in the liner.

Montmorillonite and kaolinite are important constituents of the soil in South China and are the chief available components of the geosynthetic clay liners in many landfills. Most of clays are composed of silica tetrahedral sheet(s) and alumina octahedral sheet(s). Montmorillonite, a kind of bentonite, has a 2:1 T-O-T layer structure, with the basic units consisting of two silica tetrahedral sheets and one alumina octahedral sheet in center. Interlayer swelling occurs when it is exposed to water and exchangeable cations. Kaolinite has a 1:1 TO layer structure, which is composed of units made up of a tetrahedral sheet and an octahedral sheet. However, there is no exchangeable cation, and only water molecules in the Kaolinite's interlayer. Indeed, the lack of dilatability in its interlayer results in the difficulty in exchanging reaction with substances into interlayer.

Pyrene, one of common mutagenic polycyclic aromatic hydrocarbons (PAHs) in polluted soil [12-14], is difficult to be 
decomposed in the environment $[15,16]$ and has been listed as one of sixteen kinds of PAHs priority pollutants by the US EPA. In addition, pyrene is a powerful hydrophobic substance with the $\log K_{\text {ow }}$ value of 5.18, which represents HOCs in this study.

The clay soil interactions with DOM and the mechanism of DOM-clay complexes influencing the transport of HOCs in the soil contaminated by landfill leachate are not fully understood. A major obstacle for understanding these problems is the complexity of clay soil components. Hence, montmorillonite and kaolinite, two main components of the soil in South China, were chosen to investigate the soil contaminated by landfill leachate, which provides better insight into the mechanism of the binding of DOM with clay soil and the influence of the association of DOM with clays on the sorption of HOCs.

The major mechanisms of the interactions of DOM with mineral oxide surfaces are $\mathrm{H}$-bonding, ligand exchange, van der Waals interactions, water bridging, cation bridging, and so on [17-20]. Yang et al. also suggested that humic acids adsorption by nano- $\mathrm{Al}_{2} \mathrm{O}_{3}$ could be basically explained with a ligand exchange mechanism because of the protonated $\mathrm{OH}_{2}{ }^{+}$groups on the $\mathrm{Al}_{2} \mathrm{O}_{3}$ surface [10]. Besides, many works about pyrene sorption studies have been reported. Wang et al. reported that metal oxides $\left(\mathrm{Al}_{2} \mathrm{O}_{3}, \mathrm{ZnO}\right.$, and $\mathrm{TiO}_{2}$ ) had linear isotherms for sorption of pyrene [21]. "Partitioning" like sorption is the main mechanism for the sorption of pyrene onto the bare clays. Because inorganic mineral surfaces are typically covered in aqueous solution by a layer of chemically adsorbed water molecules overlain by additional physically adsorbed water molecules, creating ice-like "vicinal" water [22]. Pyrene molecules would not be localized at specific sorption sites on oxide surfaces rather than being in the vicinal water $[21,22]$. Laegdsmand et al. who studied pyrene sorption to water-dispersible colloids under the effect of solution chemistry and organic matter found that the sorption data were described well using Freundlich isotherms and had different sorption isotherms between manured soil and depleted soil [23]. Laor et al. found that phenanthrene sorption by mineral-associated humic acid was dependent on both solution chemistry and characteristics of clays [24]. Polubesova et al. discovered that sorption and fractionation of DOM fractions by montmorillonite saturated with transition metals was important to surface controlled reactions in the retention of DOM and transport of organic colloids in soils [17]. Yang and Xing reported phenanthrene sorption by both nano- $\mathrm{TiO}_{2}$ and nano- $\mathrm{ZnO}$ was enhanced by coated humic acids [25]. However, the sorption of HOCs onto montmorillonite and kaolinite in the presence of DOM from landfill leachate has not been examined.

In this work, characterization of the bare clays and their complexes with DOM was analyzed by various techniques. Sorption experiments were performed under different contact time, initial concentrations of pyrene, and temperatures. Certain sorption kinetic models and isotherms models were applied to describe the quantitative sorption processes. These fundamental data are useful to have a better understanding of the geochemical behavior of HOCs in the soil contaminated by landfill leachate.

\section{Materials and Methods}

2.1. Materials and Reagents. The montmorillonite used in this study was obtained from a bentonite deposit in Heping (Guangdong, PRC) and the kaolinite was from a natural kaolinite mine in the Tarim Basin (Xinjiang, PRC).

DOM was extracted from the leachate of a landfill in Guangzhou (PRC) and mainly consisted of organic amine, aromatic acids, fatty acid, alcohols, phenols, and so on, as revealed by Yang and Zhou [26]. The steps of preparing DOM solution were as follows: the leachate was centrifuged for $10 \mathrm{~min}$ at $4000 \mathrm{r} \cdot \mathrm{min}^{-1}$, and the supernatant was filtered through a 0.45 um membrane, then the DOM solution was obtained and stored at $277 \mathrm{~K}$ before use. The total organic carbon (TOC) concentration of DOM solution was determined by a Shimadzu TOC-500 analyzer and its initial concentration was determined as $19.83 \mathrm{~g} \cdot \mathrm{L}^{-1}$ (almost $20000 \mathrm{ppm}$ ).

Pyrene ( $>98 \%$ purity) was purchased from Aldrich Chemical Company, USA. The molecular weight is 178.23 $\mathrm{g} \cdot \mathrm{mol}^{-1}$ and the solubility in the mixture of water and methanol $(3: 2, \mathrm{v} / \mathrm{v})$ water is $1.18 \mathrm{mg} \cdot \mathrm{L}^{-1}$ at $298 \mathrm{~K}$. Methanol is chromatogram grade and the other reagents are analytical grade. Deionized water was used in the most of sorption experiments.

Pyrene was dissolved in methanol with a typical concentration of $1000 \mathrm{mg} \cdot \mathrm{L}^{-1}$ as stock solution and then diluted into the designated concentration standards in mobile phase to construct a calibration curve. Synthetic solutions were prepared by proper dilution in deionized water. In the sorption experiments, $0.005 \mathrm{~mol} \cdot \mathrm{L}^{-1} \mathrm{CaCl}_{2}$ and $100 \mathrm{mg} \cdot \mathrm{L}^{-1}$ $\mathrm{NaN}_{3}$ of the background solution were used to prevent dissolution of sorbed organic matter and inhibit microbiological degradation of pyrene, respectively.

2.2. Preparation of the DOM-Clay Complexes. The preparation of the DOM-clay complex was as follows: First, $1.00 \mathrm{~g}$ clay was added in $100 \mathrm{~mL}$ diluted DOM solution (the ratio of solid/liquid is $1: 100$ ) under vigorous stirring for $15 \mathrm{~h}$ at $308 \mathrm{~K}$. The suspension $\mathrm{pH}$ was adjusted with $1.0 \mathrm{~mol} \cdot \mathrm{L}^{-1} \mathrm{NaOH}$ solution or $1.0 \mathrm{~mol} \cdot \mathrm{L}^{-1} \mathrm{HCl}$ solution. Then the mixture was aged for $30 \mathrm{~min}$ and centrifuged for $30 \mathrm{~min}$ at $4000 \mathrm{r} \cdot \mathrm{min}^{-1}$. The sediment was washed for 3 times with deionized water, dried at $318 \mathrm{~K}$, grounded to 200 -mush, and named as CMT or CKL.

2.3. Sorption-Desorption Experiments. Batch sorption experiments were carried out in $50 \mathrm{~mL}$ Erlenmeyer flasks by mixing together the sample (MT, CMT, KL, and CKL) with the aqueous solution of pyrene. The flasks were agitated at $170 \mathrm{r} \cdot \mathrm{min}^{-1}$ by a table concentrator, and constant temperature $\left(25 \pm 1^{\circ} \mathrm{C}\right)$ was kept with the water bath for a known time interval (30 $\mathrm{min})$. The mixture was then centrifuged at $4000 \mathrm{r} \cdot \mathrm{min}^{-1}$ for $10 \mathrm{~min}$ and the concentration of pyrene remaining in the supernatant was determined by high performance liquid chromatography (HPLC). All batch sorption experiments were replicated.

The desorption experiment was conducted as follows: After the sorption of pyrene, the suspension was centrifuged, 
and the solid was mixed with distilled water and agitated in the table concentrator [27]. The final suspension was centrifuged again $(10 \mathrm{~min})$ and the concentration of pyrene in the supernatant was determined by HPLC. In order to avoid the photodegradation of pyrene, the whole sorptiondesorption experiments were conducted in the dark. Through preliminary experiments, the volatilization and photodegradation of pyrene were negligible.

The equilibrium sorption quantity $q_{e}\left(\mu \mathrm{g} \cdot \mathrm{g}^{-1}\right)$ and the desorption percentage $D(\%)$ were determined by the following expressions:

$$
\begin{gathered}
q_{e}=\frac{V_{o}\left(C_{o}-C_{e}\right)}{1000 W_{s}}, \\
D(\%)=\frac{C_{o} C_{t} q_{e}}{1000 W_{s}} \times 100 \%,
\end{gathered}
$$

where $V_{o}(\mathrm{~mL})$ is the volume of pyrene solution and $C_{o}$ and $C_{e}\left(\mu \mathrm{g} \cdot \mathrm{g}^{-1}\right)$ are the initial concentration and equilibrium concentration, respectively. $W_{s}$ (g) denotes the mass of clay and $C_{t}$ denotes the concentration of pyrene after addition of distilled water with contact time.

\subsection{Analytical Procedure}

2.4.1. Characterization of the Materials. The X-ray diffraction (XRD) patterns of the bare clays and the DOM-clay complexes were measured with a Rigaku D/MAX-IIIA X-ray diffractometer equipped with $\mathrm{Cu} \mathrm{K} \alpha$ radiation.

The dried DOM powder and the complexes spectra were recorded by the $\mathrm{KBr}$ pellet technique on a PerkinElmer 1725X Fourier transform infrared spectrometer.

Thermogravimetry (TG) and differential scanning calorimetry (DSC) curves were obtained on a German NETZSCH TG209 and German NETZSCH DSC204C, respectively.

The microstructure at external surface of samples was observed by a Hitachi S-4500 scanning electron microscopy (SEM).

2.4.2. Reaction Solution Analysis. Pyrene was determined by high performance liquid chromatography (HPLC, Hitachi, L2000) which consisted of a UV/vis detector at $254 \mathrm{~nm}$ and a reversed-phase column of Luna $5 \mathrm{u} \mathrm{C18}$. The methanolultrapure water mixture $(90: 10)$ was used at mobile flow rate of $1.0 \mathrm{~mL} \cdot \mathrm{min}^{-1}$ under isocratic conditions at $308 \mathrm{~K}$. Samples of $20 \mu \mathrm{L}$ were injected into the column by automatic sample handling system. The relative standard deviation for HPLC analysis was controlled within $2 \%$.

\section{Results and Discussion}

\subsection{Sample Characterization}

3.1.1. X-Ray Diffraction and FTIR Spectra of Samples. The XRD results of the bare clays and their complexes are shown in Figure 1. The $d_{001}$ reflection is the main source applied to identify clays. The $d_{001}$ reflection of MT was $1.55 \mathrm{~nm}$, which represented a kind of Ca-montmorillonite [27]. The basal

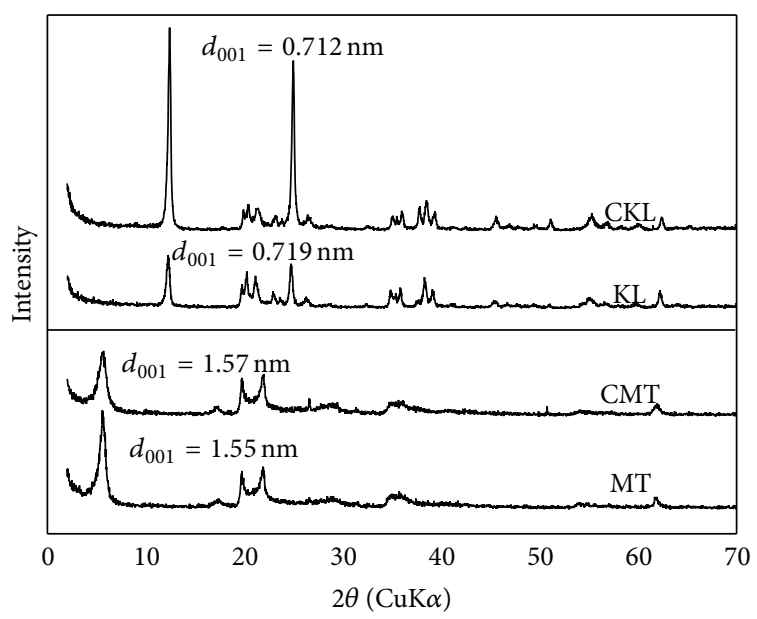

FIGURE 1: XRD patterns of MT, CMT, KL, and CKL.

spacing of CMT increased slightly to $1.57 \mathrm{~nm}$, indicating that some molecules of DOM are possibly intercalated into the interlayer. Further, the intensity was a bitweakened under the influence of DOM, which demonstrated the crystallinity decreased in the presence of DOM. A similar phenomenon was obtained by He et al. [28].

However, the XRD patterns of kaolinite in the absence and the presence of DOM were different from that of montmorillonite cases. In the XRD patterns, the $d_{001}$ reflection of CKL was sharper and a higher order harmonics appeared. This suggested that the kaolinite in the presence of DOM could be in favor of a better crystallization. In addition, the $d_{001}$ basal spacing remained basically unchanged after the introduction of DOM. No DOM molecules but water can be intercalated into the interlayer since kaolinite is the least reactive clay and lack of dilatability in its interlayer. The lack of dilatability in the interlayer of kaolinite is closely related with its structure (1:1). The two sides (oxygen and hydroxyl) of the interlayer are linked tightly by hydrogen bond, which limitsthe dilatability.

Figure 2 shows the FTIR spectra of the bare clays and their complexes. In the presence of DOM, the CMT sample exhibited an $\mathrm{OH}$ stretching which shifted from $3427 \mathrm{~cm}^{-1}$ to $3441 \mathrm{~cm}^{-1}$ and showed a lower band near $3441 \mathrm{~cm}^{-1}$. It suggested some DOM molecules were bonding with the Camontmorillonite through water bridging [17], not mechanical superposition. In comparison to MT, the CMT sample showed a lower and broader band near $1644 \mathrm{~cm}^{-1}$, which demonstrated a diminution of water in the interlayer. As shown in Figure 2, a vibration mode at $1463 \mathrm{~cm}^{-1}$, attributed to the $-\mathrm{CH}_{2}-$ or $-\mathrm{CH}_{3}$ bending mode, was observed in the spectrum of DOM, while it showed a new vibration mode at $1402 \mathrm{~cm}^{-1}$ in the spectrum of CMT. It could be inferred that some aliphatic hydrocarbon molecules from DOM were intercalated into the interlayer of montmorillonite. Similarly, Sutton and Sposito found that the DOM-montmorillonite complex featured significantly direct hydrophobic and $\mathrm{H}$ bonding interactions between organic and mineral components using molecule simulation [29]. In contrast to the 


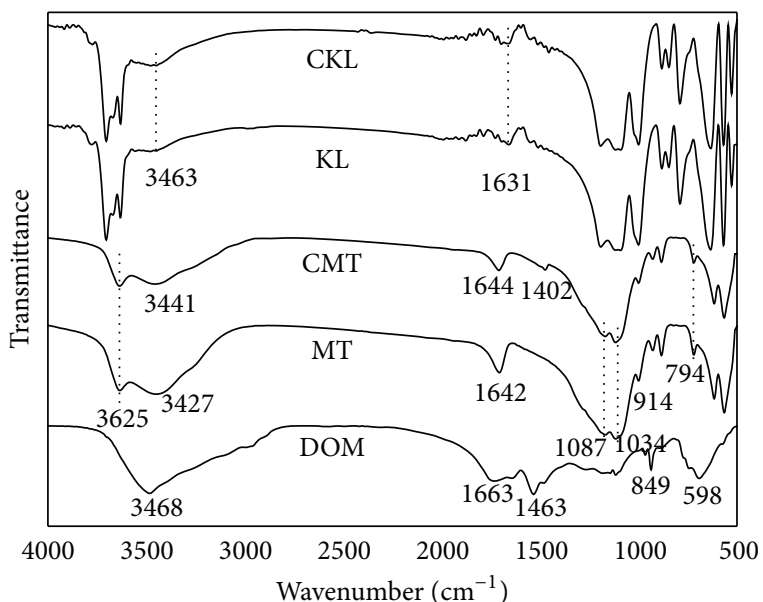

FIgURE 2: FTIR spectra of the bare clays, their complexes, and the dried DOM powder.

montmorillonite system, kaolinite is a nonexpanding clay mineral. FTIR signals of KL were identical to that of CKL, which indicated that no significant structural changes of kaolinite occurred in the absence and the presence of DOM. The result presented here agreed with those reported by Sachs and Bernhard [30], who studied the humic-kaolinite associate. In fact, the result of FTIR spectra was in accordance with the result of XRD patterns.

3.1.2. Morphology and Properties of Samples. Thermogravimetry (TG) is an important method for analyzing the thermal stability of sorbent and environments of the inserting molecules [31]. Generally, the decomposition of bare clays or organoclays consists of several steps including the following: adsorbed water and interlayer water desorption, dehydroxylation, surfactant desorption, and decomposition of clay structure [32-36]. The thermogravimetry and the differential scanning calorimetry (DSC) curves are shown in Figure 3. The MT sample showed two low-temperature decalescence peaks from 100 to $200^{\circ} \mathrm{C}$, which is mostly due to the loss of adsorbed water and interlayer water [32] and the lost weight was $15.2 \%$ (Table 1). In comparison with MT, the DSC curve of CMT became smooth and slightly straight, without sharp decalescence peaks below $200^{\circ} \mathrm{C}$. It was possible that the intercalation of DOM into the interlayer contributed to the reduction of interlayer water. This phenomenon was in agreement with the experiments performed by $\mathrm{He}$ et al. [37]. In the cases of kaolinite, there was no significant difference in TG curves in the absence and the presence of DOM. It is no more than $1 \%$ mass-loss which was assigned to the loss of humidity below $250^{\circ} \mathrm{C}$. This was related to the moisture content of kaolinite samples. The dehydroxylation of $\mathrm{KL}$ sample was observed at $525.7^{\circ} \mathrm{C}$ and that of CKL shifted a little to $529.1^{\circ} \mathrm{C}$ [38]. An exothermic valley centered at $409^{\circ} \mathrm{C}$ attributed to the loss of organic matter from the kaolinite host [39].

SEM is applied to observe the changes in morphology of the bare clays compared to the organoclays, and the results are shown in Figure 4. It was clear that MT sample had a tight and compact surface. Nevertheless, CMT sample appeared to be looser and changed into flocculent structure in the presence of DOM. The size of CMT colloids became smaller than the size of bare montmorillonite, which could significantly enhance the sorption capacity [40]. These two images (Figures 4(a) and 4(b)) present features which were similar to those previously reported for montmorillonite modified by petroleum organic compounds [39]. As shown in Figures 4(c) and 4(d), it revealed that the bare kaolin particles are clearly ball-like, and the matrix is filled with amorphous fuzzy materials [40]. However, few or no "ball" could be seen by the image of CMT sample, and the DOM-kaolinite complex transformed into network shape (Figure 4(d)). Meanwhile, this indicated a number of DOM molecules had adsorbed onto the surface of kaolinite sample. A similar phenomenon was observed for fulvic acid fractions on kaolinite [40].

A possible reaction mechanism of the interfacial interactions between DOM and clays is shown in Figure 5. In the case of montmorillonite, on one hand, a small amount of DOM molecules may be distributed in the interlayer field. Certain positively charged DOM groups could be intercalated into its interlayer through cation exchange, forming organic pillared montmorillonite, which could improve the sorption capacity. On the other hand, it was confirmed that there was an exchange of negatively charged DOM groups occurring on surface hydroxyl groups of montmorillonite silica surfaces [17], but the contribution of ligand exchange to sorption of DOM on the 2:1 layer type silicate was relatively small [41]. In contrast to the montmorillonite system, the DOM molecules were difficult to be intercalated into the interlayer field of kaolinite because the framework of interlayer is closed and no layer change is available. Thus, no DOM molecules could be intercalated into its interlayer. Nevertheless, there is an alumina octahedral sheet by exposure, which is full of hydroxyl on the surface, so carboxylate groups of DOM could interact with the alumina surface hydroxyl groups of kaolinite undergo ligand exchange [41]. Besides, the hydrophobic DOM-DOM interactions contributed to multiple adsorption of DOM on clays surfaces and went against the Langmuir model.

\subsection{Sorption-Desorption Experiments}

3.2.1. Sorption Kinetics. To test the rate of the sorption process and investigate the potential rate-controlling step, a series of sorption experiments at constant temperature was performed, the adsorptive capacity $\left(q_{t}\right)$ was monitored, and several kinetic models were investigated.

The sorption kinetics was tested by the following famous models, processed by nonlinear least squares fitting [42, 43] (NLSF) using data analysis and graphing software Origin Pro 8 (OriginLab Corporation, USA).

(a) Elovish equation $[44,45]$ is

$$
q_{t}=\frac{1}{b} \ln (a b)+\frac{1}{b} \ln t
$$




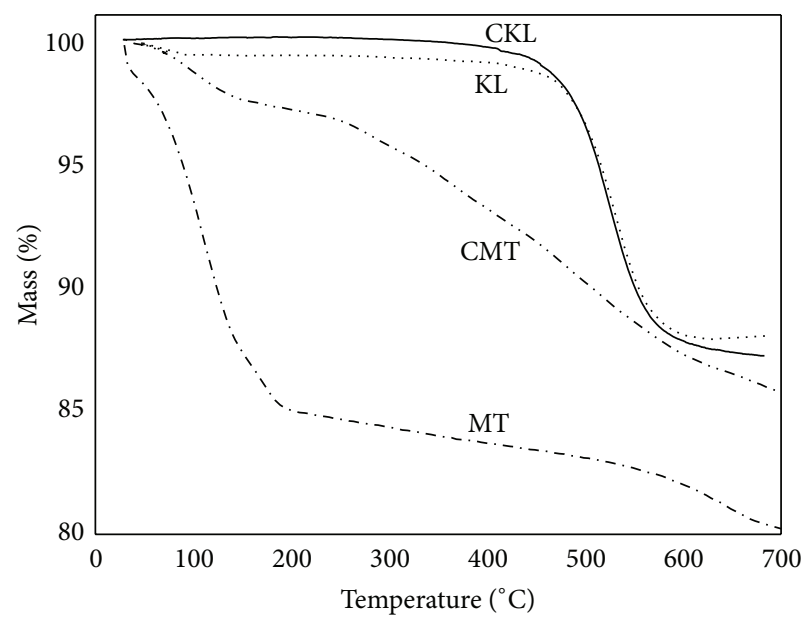

(a)

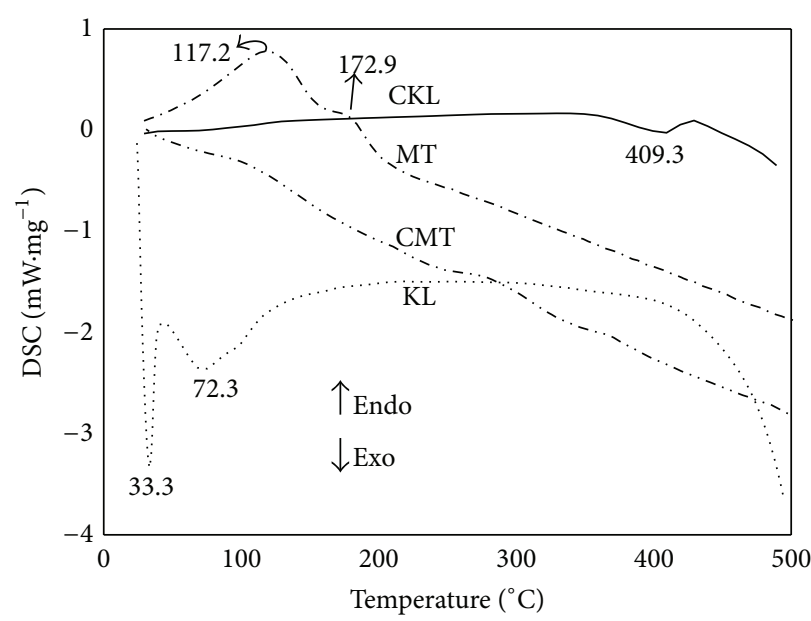

(b)

FIGURE 3: TG (a) and DSC (b) curves of MT, CMT, KL, and CKL.

TABLE 1: TG results of samples (MT, CMT, KL, and CKL).

\begin{tabular}{lccc}
\hline Samples & $\begin{array}{c}\text { Temperature } \\
\left({ }^{\circ} \mathrm{C}\right)\end{array}$ & $\begin{array}{c}\text { Mass loss } \\
(\%)\end{array}$ & Reasons \\
\hline CMT & 179.0 & 2.7 & Loss of humidity \\
MT & 172.9 & 15.2 & $\begin{array}{c}\text { Loss of humidity } \\
\text { Dehydroxylation and loss } \\
\text { CKL }\end{array}$ \\
of organic matter \\
KL & 525.7 & 12.9 & Dehydroxylation \\
\hline
\end{tabular}

where $t$ is sorption time, $q_{t}$ is the amounts sorbed per unit mass with time $t$, and $a$ and $b$ are sorption constant.

(b) Pseudo-first-order model $[46,47]$ is

$$
q_{t}=q_{e}\left[1-\exp \left(-k_{1} t\right)\right]
$$

where $q_{e}$ is the amounts sorbed per unit mass at equilibrium time and $k_{1}$ is the pseudo-first-order sorption rate constant.

(c) Pseudo-second-order model $[46,47]$ is

$$
q_{t}=\frac{q_{e}^{2} k_{2} t}{1+q_{e} k_{2} t},
$$

where $k_{2}$ is the pseudo-first-order sorption rate constant. $q_{e}^{2} k_{2}$ is described as the initial sorption rate as $t$ to 0 . This model is in agreement with chemical sorption being the rate-controlling step [48].

The amount of pyrene sorbed on unit mass of samples $\left(q_{t}\right)$ for various contact times at the initial concentration of $1000 \pm 150 \mu \mathrm{g} \cdot \mathrm{L}^{-1}$ are shown in Figure 6. The initial $\mathrm{pH}$ before sorption was 7.3. A little change of $\mathrm{pH}$ was observed after equilibrium in the preliminary experiment. At the beginning, the sorption of pyrene proceeded at a rapid rate as a large number of sorption sites were available. As the sorption sites were filled up gradually, sorption proceeded slowly and the kinetics became more dependent on the rate at which the sorption was transferred from the bulk phase to the actual sorption sites [49]. The efficiency of sorption was high with 20 min enough to nearly reach equilibrium and equilibrium was achieved after $30 \mathrm{~min}$. Indeed, the sorption rate by MT was a bit slower than others in the first $20 \mathrm{~min}$. The sorption capacity of CMT was nearly twice higher than MT, suggesting that DOM-montmorillonite complex was favorable toward promoting the sorption of pyrene. The fact that the sorption capacity of KL was obviously higher than MT may be related to the difference in hydrophobic nature of sorbents. There is substitution of $\mathrm{Al}^{3+}$ by $\mathrm{Mg}^{2+}$ in the octahedral sheet and of $\mathrm{Si}^{4+}$ by $\mathrm{Al}^{3+}$ in the tetrahedral sheet, which results in negative net charge in the interlayer of montmorillonite. However, there is no substitution in the layer of kaolinite, indicating that the net layer charge of kaolinite is zero. Therefore, the silica surface of bare kaolinite has stronger hydrophobic nature than that of bare montmorillonite. In the case of kaolinite, although many potential sorption sites on the bare kaolinite surface were reduced by the DOM coating, new sorption sites would be produced subsequently. As shown in Figure 6, there was minimal difference concerning sorption capacity between CKL and KL, indicating that the contribution of the new sorption sites for sorption capacity was nearly equivalent to the hydrophobicity of bare kaolinite.

The fitting results of kinetics models, including the experimental and calculated $q_{e}$ values, rate constants $(k)$, and coefficient of correlation coefficients $\left(R^{2}\right)$, are given in Table 1 . Obviously, the correlation coefficients for the Elovich model obtained by CMT, CKL, and KL were very low. It suggested that the sorption of pyrene did not follow Elovich model.

The pseudo-first-order mode had correlation coefficients $\left(R^{2}\right)$ in the range of 0.97 to 0.99 and the calculated $q_{e}$ values agree well with the experimental $q_{e}$ (Table 2). The pseudo-first-order sorption rate $\left(k_{1}\right)$ lies between 0.61 and $1.32 \mathrm{~min}^{-1} . K_{f}$ values were in the order of CMT $>\mathrm{KL}>\mathrm{CKL}>$ MT. The kinetic data were also tested with respect to the 


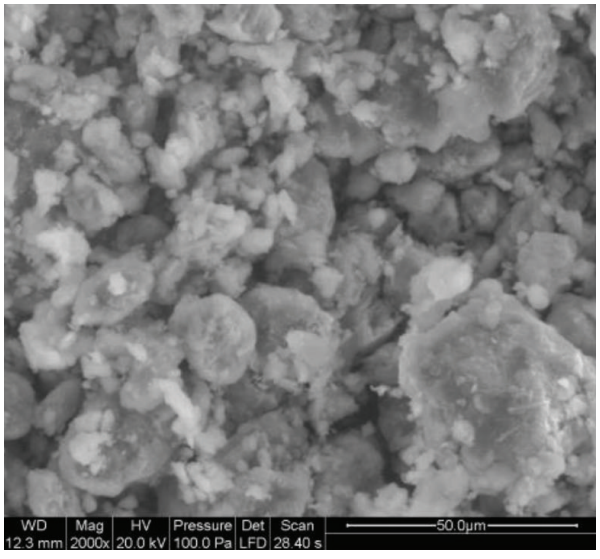

(a)

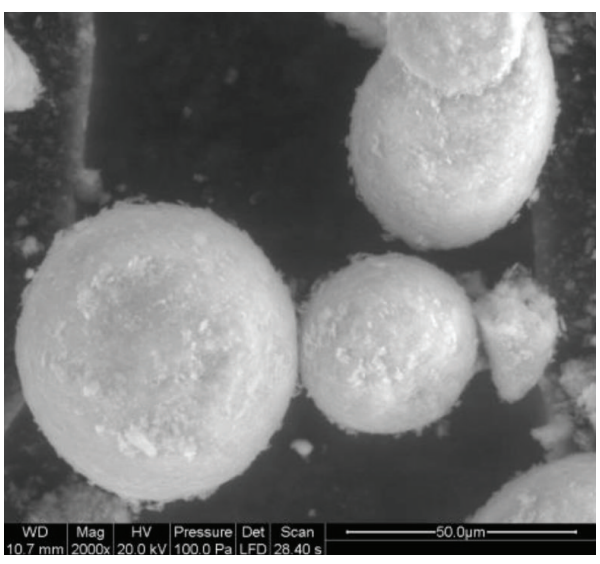

(c)

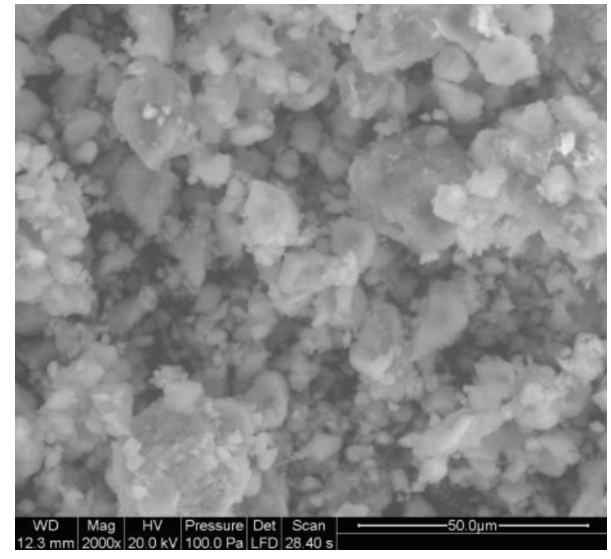

(b)

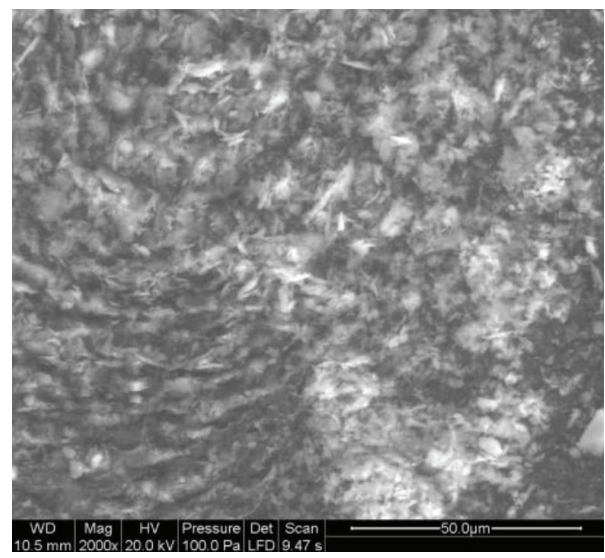

(d)

Figure 4: SEM images of MT (a), CMT (b), KL (c), and CKL (d).

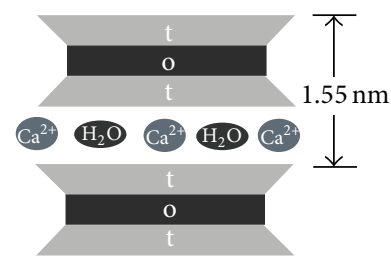

Montmorillonite (MT)

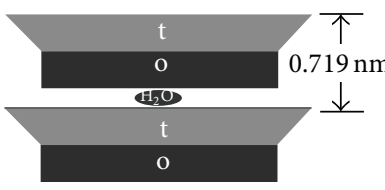

Kaolinite (KL)

o: octahedral sheets $\mathrm{t}$ : tetrahedral sheets

FIGURE 5: Schematic representation of the interfacial interactions between clays and DOM. 
TABLE 2: The coefficients of the kinetic models for sorption of pyrene at $298 \mathrm{~K}$.

\begin{tabular}{lcccccccc}
\hline \multirow{2}{*}{ Sample } & Elovich & \multicolumn{3}{c}{ Pseudo-first-order } & \multicolumn{3}{c}{ Pseudo-second-order } & Experimental \\
& $R^{2}$ & $q_{e}$ & $k_{1}$ & $R^{2}$ & $q_{e}$ & $k_{2} \times 10^{3}$ & $R^{2}$ & $q_{e}$ \\
\hline CMT & 0.152 & 38.63 & 1.32 & 0.98 & 38.69 & 4.18 & 0.99 & 38.96 \\
MT & 0.929 & 21.76 & 0.61 & 0.97 & 22.01 & 25.97 & 0.98 & 22.18 \\
CKL & 0.792 & 44.56 & 0.85 & 0.99 & 44.72 & 7.04 & 0.99 & 44.99 \\
KL & 0.774 & 41.95 & 1.01 & 0.98 & 42.01 & 4.86 & 0.99 & 42.00 \\
\hline
\end{tabular}

Note. The units of $k_{1}$ and $k_{2}$ are, respectively, $\min ^{-1}$ and $\mathrm{g} \cdot \mu \mathrm{g}^{-1} \mathrm{~min}^{-1}$.

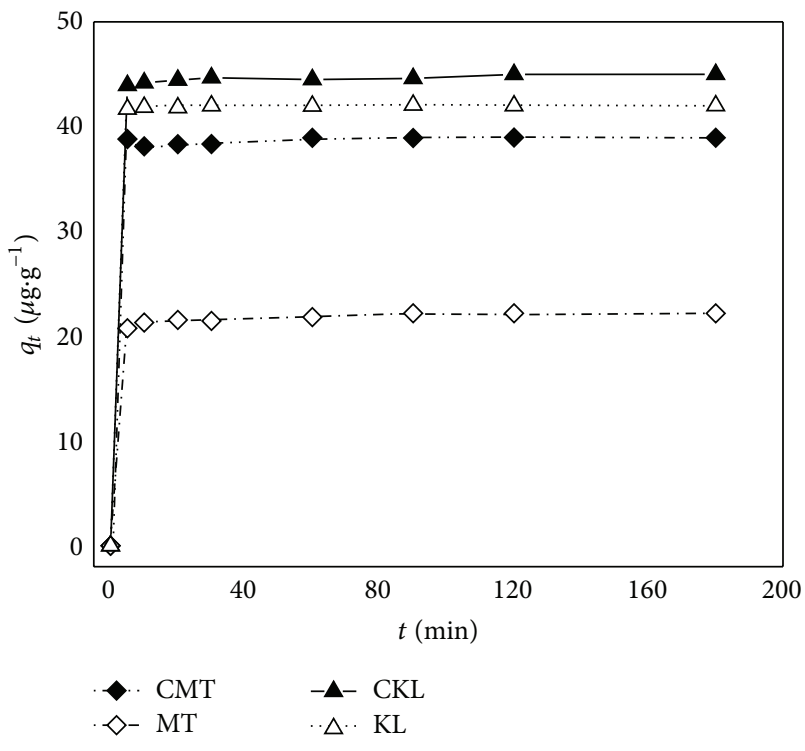

FIGURE 6: Effect of time on the sorption of pyrene by the bare clays and their complexes at $298 \mathrm{~K}$.

pseudo-second-order model, as high value of the correlation coefficients and the calculated $q_{e}$ values agreed well with the experimental $q_{e}$. Therefore, both models (pseudo-firstorder and second-order) provided a good description of the sorption process; thus, the sorption can be described as a chemical-reaction-based process. A similar result was obtained by Valderrama et al. [50].

3.2.2. Sorption Isotherms. Sorption isotherms are used to indicate the partition of adsorbate between solution and adsorbent at equilibrium [47]. Sorption isotherms were studied by operating a set of sorption experiments at constant temperature with different equilibrium concentrations of the adsorbate. The sorption isotherm was tested by the following models using NLSF.

(i) Henry isotherm, is usually used to describe the linear sorption process and its formula is

$$
q_{e}=K_{D} C_{e}
$$

where $C_{e}$ and $q_{e}$ are the equilibrium concentrations of the adsorbate in the liquid and the solid phases, respectively; the $K_{D}$ is the sorption distribution coefficient. (ii) Freundlich isotherm, is usually used to describe nonhomogeneous surface sorption process (an empirical sorption model) and its formula is

$$
q_{e}=K_{f} C_{e}^{n},
$$

where $K_{f}$ is Freundlich sorption coefficient, relating to the capacity and the intensity of sorption and $n$ is Freundlich sorption index.

For a series of initial concentrations of pyrene from $100 \mu \mathrm{g} \cdot \mathrm{L}^{-1}$ to $1000 \mu \mathrm{g} \cdot \mathrm{L}^{-1}$, the $q_{e}$ versus $C_{e}$ isotherms for pyrene-samples interactions with a fixed adsorbent amount $20 \mathrm{~g} \cdot \mathrm{L}^{-1}$ at $298 \mathrm{~K}$ are shown in Figure 7 . It was observed that the sorption isotherms of CKL and CMT had a similar feature. Obviously, CMT, CKL, and KL reached the equilibrium amount in solid phase at much lower equilibrium concentration in liquid phase than that of MT. In addition, the CMT, CKL, and KL had much higher uptake of pyrene compared to that of MT. Accordingly, saturation of sorption sites on MT had been attained with a much lower concentration of pyrene and the others with a much higher concentration of pyrene [51].

The sorption data were analyzed by Henry and Freundlich models. The correlation coefficients $\left(R^{2}\right)$ and other parameters are summarized in Table 3. Both the Henry isotherm and the Freundlich isotherm showed good fits to the isotherms data. Indeed, the sorption isotherms by the bare clays showed a relatively high value of $R^{2}$ for Henry model, whereas Freundlich model provided a better fit to the equilibrium data of sorption onto the complexes. Furthermore, the sorption intensity given by the Freundlich sorption index, $n$, approached 1.0 in the cases of bare clays, which further indicated the process of the sorption onto the bare clays well followed the linear model. The DOM molecules adsorbed onto clays contributed to the mineral surfaces covered by a layer of hydrophobic substance which contained aromatic content. The sorption of pyrene was likely related to this aromatic content on the complexes' surfaces [52]. Besides, the role of the retention of pyrene on the complexes surfaces is not only dispersion, but also important of $\pi-\pi$ interactions [50].

3.2.3. Effect of the Different Temperatures. The temperature of the aqueous solution is a fundamental parameter in the sorption process. Figure 8 represents the effect of temperature on the equilibrium amount of pyrene sorbed on unit mass 
TABLE 3: Parameters of Henry isotherm and Freundlich isotherm at $298 \mathrm{~K}$.

\begin{tabular}{lccccc}
\hline \multirow{2}{*}{ Sorbents } & \multicolumn{2}{c}{ Henry } & \multicolumn{3}{c}{ Freundlich } \\
& $K_{D}$ & $R^{2}$ & $K_{f} \times 10^{3}$ & $n$ & $R^{2}$ \\
\hline CMT & 0.28 & 0.90 & 1.69 & 2.13 & 0.94 \\
MT & 0.16 & 0.98 & 30.67 & 1.33 & 0.96 \\
CKL & 0.26 & 0.88 & 0.16 & 2.61 & 0.92 \\
KL & 0.18 & 0.95 & 15.93 & 1.49 & 0.90 \\
\hline Note. The units of $K_{D}$ and $K_{f}$ are, respectively, $\mathrm{L}^{-1}$ and $\mu \mathrm{g}^{(1-1 / n)} \cdot \mathrm{L}^{1 / n} \cdot \mathrm{g}^{-1}$.
\end{tabular}

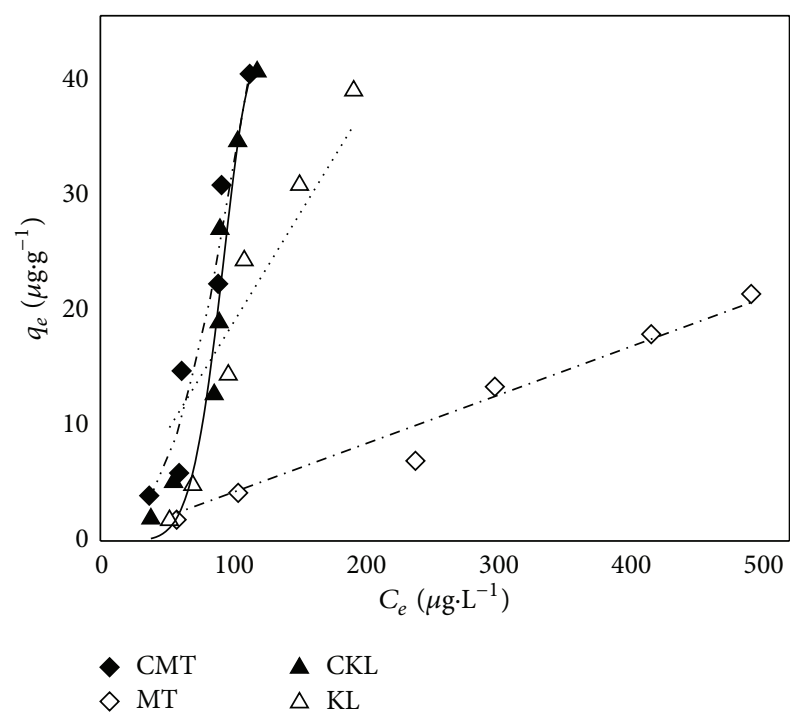

FIGURE 7: Isothermal sorption plot of pyrene by the bare clays and their complexes at $298 \mathrm{~K}$.

of sorbents $\left(q_{e}\right)$. The reaction temperature had a significant effect on the sorption, especially when sorbents were the complexes. Importantly, this figure revealed that the amount of pyrene sorbed onto the sorbents per unit mass of sorbents decreased as the temperature of the process increased from 298 to $318 \mathrm{~K}$. Accordingly, pyrene sorbed onto CMT, MT, $\mathrm{CKL}$, and $\mathrm{KL}$ was an exothermal process in the range from $298 \mathrm{~K}$ to $318 \mathrm{~K}$.

3.2.4. Desorption of Pyrene. In order to test the stability of sorbents after sorption, desorption experiment was conducted after sorption experiment at $298 \mathrm{~K}$. Figure 9 shows the trend in desorption percentage of pyrene with different contact time. Desorption percentage was obtained by determining the rate of the amount of pyrene stripping from unit mass of samples to equilibrium sorption quantity $\left(q_{e}\right)$. Desorption results showed that very little amounts of pyrene were desorbed from CMT, which indicated the binding of pyrene with the DOM-montmorillonite complex were strong [27]. Nevertheless, almost the same percentage of pyrene desorbed from KL and CKL, suggesting DOM had little effect on the binding of pyrene with kaolinite.

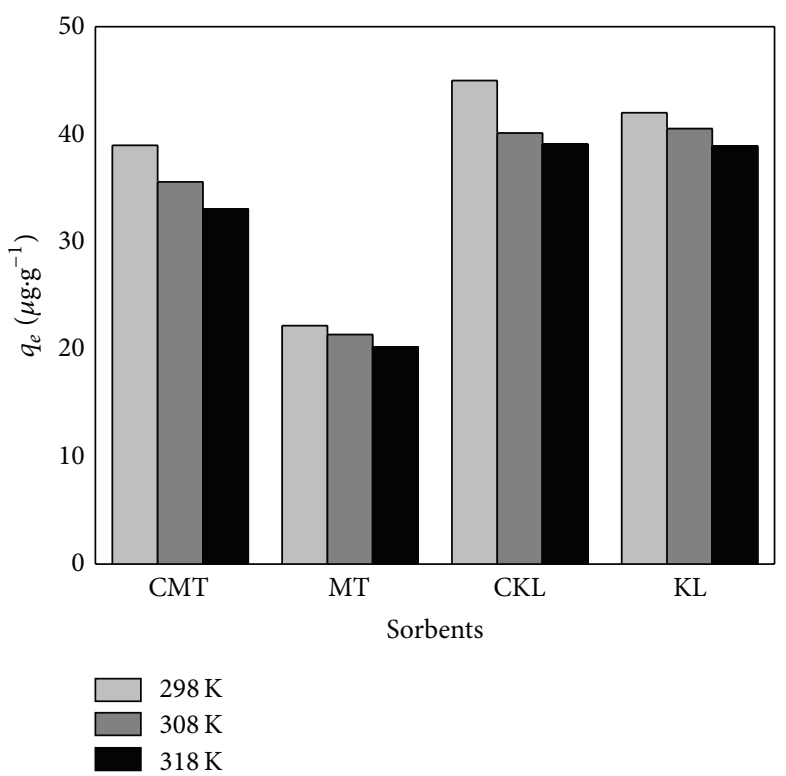

FIgURE 8: Effect of temperature on sorption capacity.

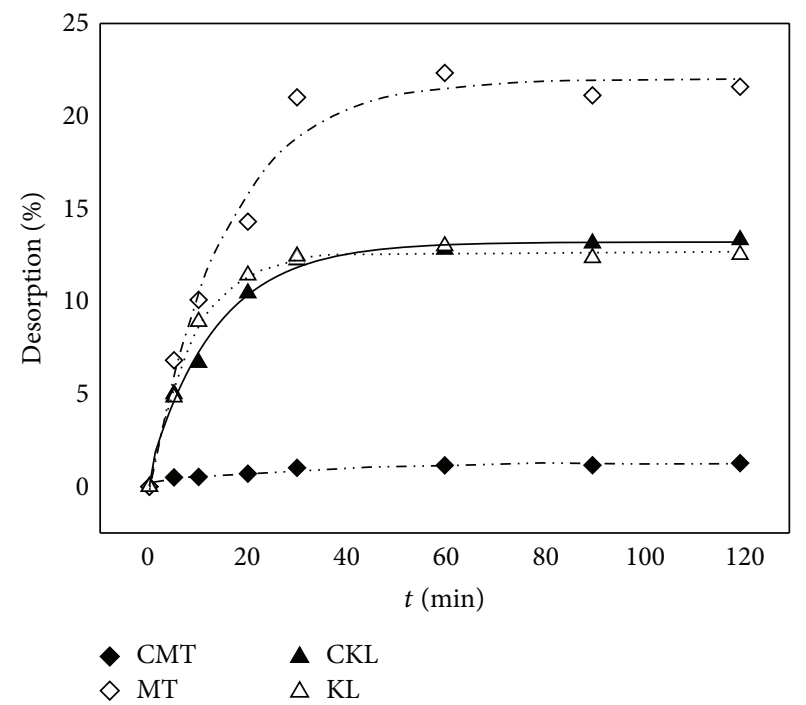

FIGURE 9: Desorption plot of pyrene from the bare clays and their complexes at $298 \mathrm{~K}$.

\section{Conclusion}

In this study, the interactions of DOM from landfill leachate with the clays were discussed and the sorption of pyrene onto the clays in the presence of DOM was investigated. The results of characterization indicated that the adsorption of DOM mainly occurred on the flat exposed planes of the silica and alumina sheets. A small amount of DOM molecules may be intercalated into the interlayer of montmorillonite, but no DOM molecules were intercalated into the interlayer of kaolinite. The changes in morphology of the clays surfaces were strongly affected by DOM, and especially the morphology of kaolinite particles transformed into network shape. 
The results of sorption-desorption experiments indicated the sorption isotherms of pyrene by the bare clays followed the Henry model well, whereas the isotherms by their complexes with DOM were well described by the Freundlich model. Association of DOM with montmorillonite would remarkably reduce the mobility of HOCs in the soil contaminated by landfill leachate than the bare ones. However, association of DOM with kaolinite did not affect significantly the retention of pyrene in comparison to the bare kaolinite. Therefore, montmorillonite played a more dominant role in the persistence of HOCs in the soil contaminated by landfill leachate than kaolinite.

\section{Conflict of Interests}

The authors declare that there is no conflict of interests regarding the publication of this paper.

\section{Acknowledgments}

The authors are grateful for financial support from the National Science Foundation of China (Grants no. 40973075, 41073058, 41273122) and Research Fund for the Doctoral Program of Higher Education of China (no. 20100172110028).

\section{References}

[1] A. Goi, Y. Veressinina, and M. Trapido, "Fenton process for landfill leachate treatment: evaluation of biodegradability and toxicity," ASCE Journal of Environmental Engineering, vol. 136, no. 1, pp. 46-53, 2010.

[2] D. J. L. Thomas, S. F. Tyrrel, R. Smith, and S. Farrow, "Bioassays for the evaluation of landfill leachate toxicity," Journal of Toxicology and Environmental Health B, vol. 12, pp. 83-105, 2009.

[3] J. Olivero-Verbel, C. Padilla-Bottet, and O. de la Rosa, "Relationships between physicochemical parameters and the toxicity of leachates from a municipal solid waste landfill," Ecotoxicology and Environmental Safety, vol. 70, no. 2, pp. 294-299, 2008.

[4] P.-J. He, J.-F. Xue, L.-M. Shao, G.-J. Li, and D.-J. Lee, "Dissolved organic matter (DOM) in recycled leachate of bioreactor landfill," Water Research, vol. 40, no. 7, pp. 1465-1473, 2006.

[5] N. Calace, A. Liberatori, B. M. Petronio, and M. Pietroletti, "Characteristics of different molecular weight fractions of organic matter in landfill leachate and their role in soil sorption of heavy metals," Environmental Pollution, vol. 113, no. 3, pp. 331339, 2001

[6] D. C. Smith, J. Sacks, and E. Senior, "Irrigation of soil with synthetic landfill leachate-speciation and distribution of selected pollutants," Environmental Pollution, vol. 106, no. 3, pp. 429441, 1999.

[7] D. C. Smith, E. Senior, and H. M. Dicks, "Irrigation of soil with synthetic landfill leachate-breakthrough behaviour of selected pollutants," Water, Air, and Soil Pollution, vol. 109, no. 1-4, pp. 327-342, 1999.

[8] J. Antelo, F. Arce, M. Avena, S. Fiol, R. López, and F. Macías, "Adsorption of a soil humic acid at the surface of goethite and its competitive interaction with phosphate," Geoderma, vol. 138, no. 1-2, pp. 12-19, 2007.
[9] G. Guggenberger and K. Kaiser, "Dissolved organic matter in soil: challenging the paradigm of sorptive preservation," Geoderma, vol. 113, no. 3-4, pp. 293-310, 2003.

[10] K. Yang, L. Z. Zhu, and B. Xing, "Sorption of phenanthrene by nanosized alumina coated with sequentially extracted humic acids," Environmental Science and Pollution Research, vol. 17, no. 2, pp. 410-419, 2010.

[11] A. A. Roberts and T. Shimaoka, "Analytical study on the suitability of using bentonite coated gravel as a landfill liner material," Waste Management, vol. 28, no. 12, pp. 2635-2644, 2008.

[12] T. Chafik, "Evaluation of the soil contamination of tangier (Morocco) by the determination of BTEX, PCBs, and PAHs," Soil and Sediment Contamination, vol. 18, no. 6, p. 766, 2009.

[13] J. A. O. Cotta, M. O. O. Rezende, and M. D. Landgraf, "Evaluation of solvent extraction by ultrasound by using high performance liquid chromatography for the determination of polycyclic aromatic hydrocarbons in contaminated soils," Química Nova, vol. 32, no. 8, pp. 2026-2033, 2009.

[14] Y. Teng, Y. Luo, L. Ping, D. Zou, Z. Li, and P. Christie, "Effects of soil amendment with different carbon sources and other factors on the bioremediation of an aged PAH-contaminated soil," Biodegradation, vol. 21, no. 2, pp. 167-178, 2010.

[15] K. Gondek, M. Kopec, M. Chmiel, and I. Spalek, "Response of zea maize and microorganisms to soil pollution with polycyclic aromatic hydrocarbons (PAHs)," Polish Journal of Environmental Studies, vol. 17, no. 6, pp. 875-880, 2008.

[16] S. Tamamura, T. Sato, Y. Ota, N. Tang, and K. Hayakawa, "Decomposition of polycyclic aromatic hydrocarbon (PAHs) on mineral surface under controlled relative humidity," Acta Geologica Sinica, vol. 80, no. 2, pp. 185-191, 2006.

[17] T. Polubesova, Y. Chen, R. Navon, and B. Chefetz, "Interactions of hydrophobic fractions of dissolved organic matter with $\mathrm{Fe}^{3+}$ - and $\mathrm{Cu}^{2+}$-montmorillonite," Environmental Science and Technology, vol. 42, no. 13, pp. 4797-4803, 2008.

[18] B. Gu, J. Schmitt, Z. Chen, L. Liang, and J. F. McCarthy, "Adsorption and desorption of natural organic matter on iron oxide: mechanisms and models," Environmental Science and Technology, vol. 28, no. 1, pp. 38-46, 1994.

[19] D. Vasudevan and A. T. Stone, "Adsorption of catechols, 2-aminophenols, and 1,2-phenylenediamines at the metal (hydr)oxide/water interface: effect of ring substituents on the adsorption onto $\mathrm{TiO}_{2}$," Environmental Science and Technology, vol. 30, no. 5, pp. 1604-1613, 1996.

[20] F. Elfarissi and E. Pefferkorn, "Kaolinite/humic acid interaction in the presence of aluminium ion," Colloids and Surfaces, vol. 168 , no. 1, pp. 1-12, 2000.

[21] X. L. Wang, J. L. Lu, M. G. Xu, and B. S. Xing, "Sorption of pyrene by regular and nanoscaled metal oxide particles: influence of adsorbed organic matter," Environmental Science and Technology, vol. 42, no. 19, pp. 7267-7272, 2008.

[22] W. L. Huang, M. A. Schlautman, and W. J. Weber Jr., "A distributed reactivity model for sorption by soils and sediments. 5. The influence of near-surface characteristics in mineral domains," Environmental Science and Technology, vol. 30, no. 10, pp. 2993-3000, 1996.

[23] M. Laegdsmand, L. W. de Jonge, P. Moldrup, and K. Keiding, "Pyrene sorption to water-dispersible colloids: effect of solution chemistry and organic matter," Vadose Zone Journal, vol. 3, no. 2, pp. 451-461, 2004. 
[24] Y. Laor, W. J. Farmer, Y. Aochi, and P. F. Strom, "Phenanthrene binding and sorption to dissolved and to mineral-associated humic acid," Water Research, vol. 32, no. 6, pp. 1923-1931, 1998.

[25] K. Yang and B. S. Xing, "Sorption of phenanthrene by humic acid-coated nanosized $\mathrm{TiO}_{2}$ and $\mathrm{ZnO}$," Environmental Science \& Technology, vol. 43, no. 6, pp. 1845-1851, 2009.

[26] Z. Yang and S. Zhou, "The biological treatment of landfill leachate using a simultaneous aerobic and anaerobic (SAA) bio-reactor system," Chemosphere, vol. 72, no. 11, pp. 1751-1756, 2008.

[27] S. Z. Li and P. X. Wu, "Characterization of sodium dodecyl sulfate modified iron pillared montmorillonite and its application for the removal of aqueous $\mathrm{Cu}(\mathrm{II})$ and $\mathrm{Co}(\mathrm{II})$," Journal of Hazardous Materials, vol. 173, pp. 62-70, 2010.

[28] H. P. He, D. Yang, P. Yuan, W. Shen, and R. L. Frost, "A novel organoclay with antibacterial activity prepared from montmorillonite and Chlorhexidini Acetas," Journal of Colloid and Interface Science, vol. 297, no. 1, pp. 235-243, 2006.

[29] R. Sutton and G. Sposito, "Molecular simulation of humic substance-Ca-montmorillonite complexes," Geochimica et Cosmochimica Acta, vol. 70, pp. 3566-3581, 2006.

[30] S. Sachs and G. Bernhard, "Sorption of U(VI) onto an artificial humic substance-kaolinite-associate," Chemosphere, vol. 72, no. 10, pp. 1441-1447, 2008.

[31] L. Zhou, H. Chen, X. Jiang et al., "Modification of montmorillonite surfaces using a novel class of cationic gemini surfactants," Journal of Colloid and Interface Science, vol. 332, no. 1, pp. 16-21, 2009.

[32] P. X. Wu, H. H. Wu, and R. Li, "The microstructural study of thermal treatment montmorillonite from Heping, China," Spectrochimica Acta A, vol. 61, pp. 3020-3025, 2005.

[33] Y. F. Xi, R. L. Frost, H. He, T. Kloprogge, and T. Bostrom, "Modification of Wyoming montmorillonite surfaces using a cationic surfactant," Langmuir, vol. 21, no. 19, pp. 8675-8680, 2005.

[34] Y. F. Xi, Q. Zhou, R. L. Frost, and H. P. He, "Thermal stability of octadecyltrimethylammonium bromide modified montmorillonite organoclay," Journal of Colloid and Interface Science, vol. 311, no. 2, pp. 347-353, 2007.

[35] S. I. Marras, A. Tsimpliaraki, I. Zuburtikudis, and C. Panayiotou, "Thermal and colloidal behavior of amine-treated clays: the role of amphiphilic organic cation concentration," Journal of Colloid and Interface Science, vol. 315, no. 2, pp. 520-527, 2007.

[36] R. R. Tiwari, K. C. Khilar, and U. Natarajan, "Synthesis and characterization of novel organo-montmorillonites," Applied Clay Science, vol. 38, no. 3-4, pp. 203-208, 2008.

[37] H. He, J. Duchet, J. Galy, and J.-F. Gérard, "Influence of cationic surfactant removal on the thermal stability of organoclays," Journal of Colloid and Interface Science, vol. 295, no. 1, pp. 202208, 2006.

[38] T. A. Elbokl and C. Detellier, "Intercalation of cyclic imides in kaolinite," Journal of Colloid and Interface Science, vol. 323, no. 2, pp. 338-348, 2008.

[39] A. Cosultchi, P. Bosch, and V. H. Lara, "Adsorption of petroleum organic compounds on natural Wyoming montmorillonite," Colloids and Surfaces A: Physicochemical and Engineering Aspects, vol. 243, no. 1-3, pp. 53-61, 2004.

[40] A. M. Li, M. J. Xu, W. H. Li, X. J. Wang, and J. Y. Dai, "Adsorption characterizations of fulvic acid fractions onto kaolinite," Journal of Environmental Sciences, vol. 20, pp. 528-535, 2008.
[41] J. Chorover and M. K. Amistadi, "Reaction of forest floor organic matter at goethite, birnessite and smectite surfaces," Geochimica et Cosmochimica Acta, vol. 65, no. 1, pp. 95-109, 2001.

[42] T. Perger, T. Kovacs, T. Turanyi, and C. Trevino, "Determination of the adsorption and desorption parameters for ethene and propene from measurements of the heterogeneous ignition temperature," Combustion and Flame, vol. 142, p. 107, 2005.

[43] H. D. Yoo, J. H. Jang, B. H. Ka, C. K. Rhee, and S. M. Oh, "Impedance analysis for hydrogen adsorption pseudocapacitance and Electrochemically active surface area of Pt electrode," Langmuir, vol. 25, no. 19, pp. 11947-11954, 2009.

[44] R. L. Tseng, F. C. Wu, and R. S. Juang, "Liquid-phase adsorption of dyes and phenols using pinewood-based activated carbons," Carbon, vol. 41, no. 3, pp. 487-495, 2003.

[45] Y. S. Ho, "Review of second-order models for adsorption systems," Journal of Hazardous Materials, vol. 136, no. 3, pp. 681689, 2006.

[46] X. Y. Yang and B. Al-Duri, "Kinetic modeling of liquidphase adsorption of reactive dyes on activated carbon," Journal of Colloid and Interface Science, vol. 287, pp. 25-34, 2005.

[47] Y. Wang, B.-Y. Gao, W.-W. Yue, and Q.-Y. Yue, "Adsorption kinetics of nitrate from aqueous solutions onto modified wheat residue," Colloids and Surfaces A, vol. 308, no. 1-3, pp. 1-5, 2007.

[48] Y. S. Ho and G. McKay, "A kinetic study of dye sorption by biosorbent waste product pith," Resources, Conservation and Recycling, vol. 25, pp. 171-193, 1999.

[49] B. Yu, Y. Zhang, A. Shukla, S. S. Shukla, and K. L. Dorris, "The removal of heavy metal from aqueous solutions by sawdust adsorption-removal of copper," Journal of Hazardous Materials, vol. 80, no. 1-3, pp. 33-42, 2000.

[50] C. Valderrama, J. L. Cortina, A. Farran, X. Gamisans, and C. Lao, "Kinetics of sorption of polyaromatic hydrocarbons onto granular activated carbon and Macronet hyper-cross-linked polymers (MN200)," Journal of Colloid and Interface Science, vol. 310, no. 1, pp. 35-46, 2007.

[51] K. G. Bhattacharyya and S. Sen Gupta, "Influence of acid activation of kaolinite and montmorillonite on their adsorptive removal of Cd(II) from water," Industrial \& Engineering Chemistry Research, vol. 4, pp. 3734-3742, 2007.

[52] C. T. Chiou, S. E. McGroddy, and D. E. Kile, "Partition characteristics of polycyclic aromatic hydrocarbons on soils and sediments," Environmental Science and Technology, vol. 32, pp. 264-269, 1998. 

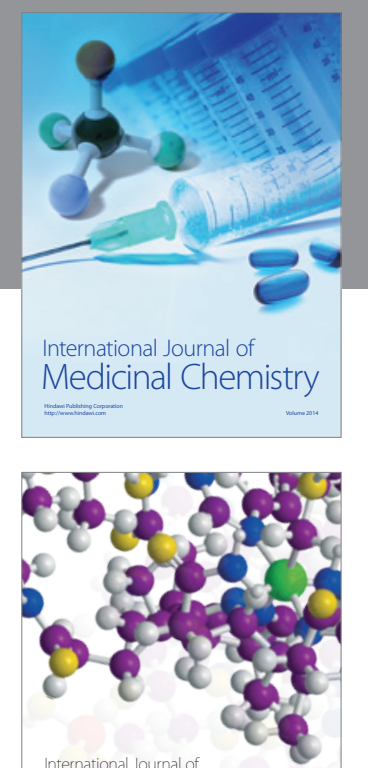

\section{Carbohydrate} Chemistry

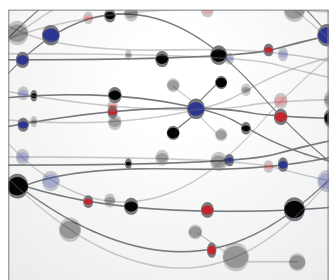

The Scientific World Journal
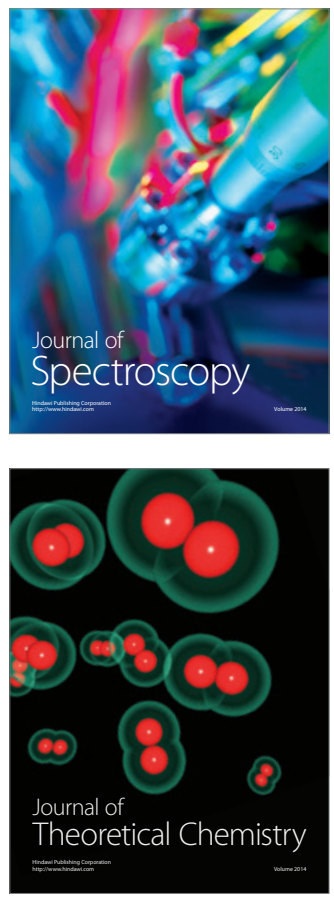
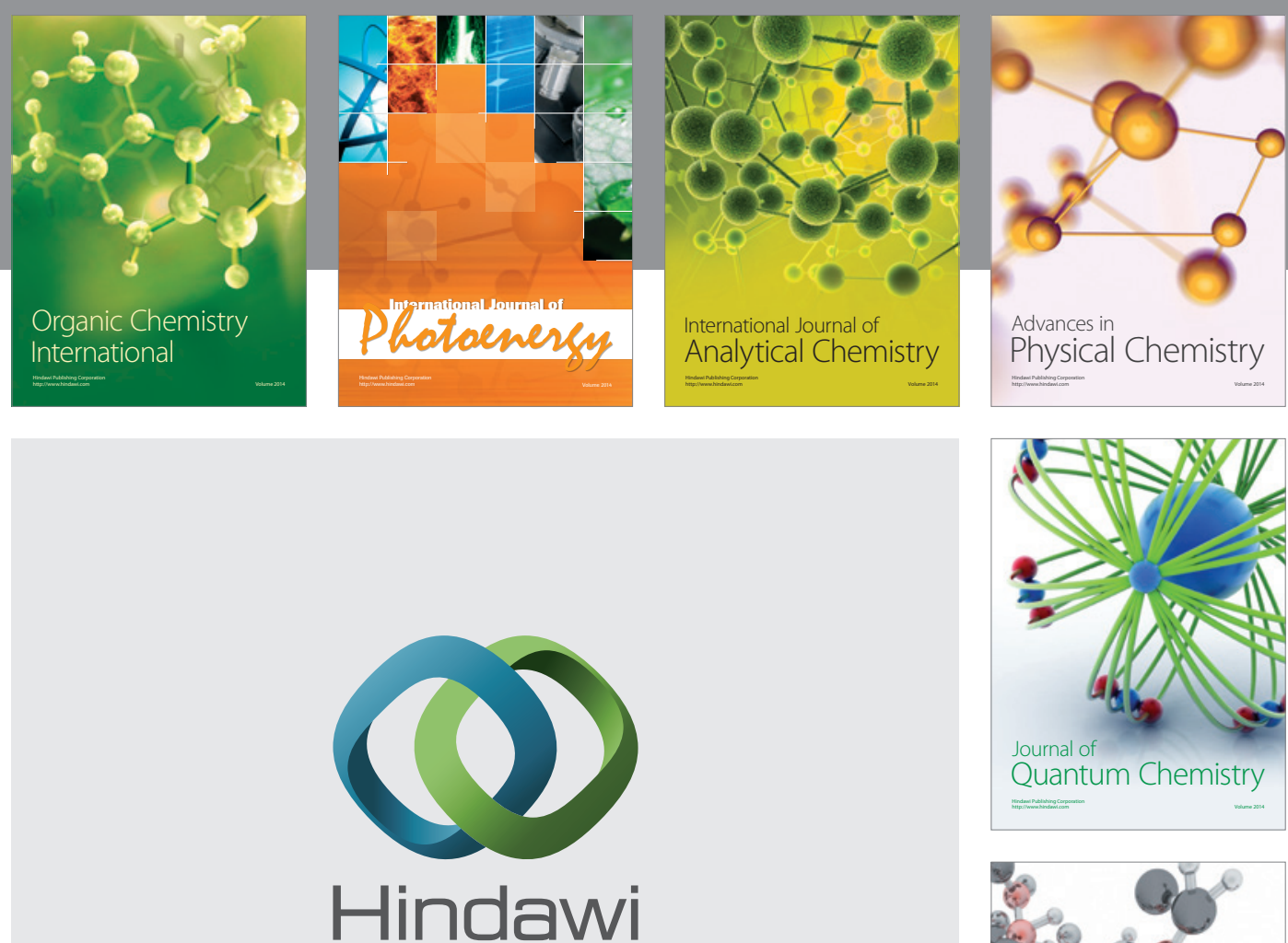

Submit your manuscripts at

http://www.hindawi.com

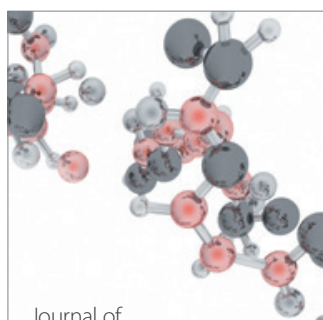

Analytical Methods

in Chemistry

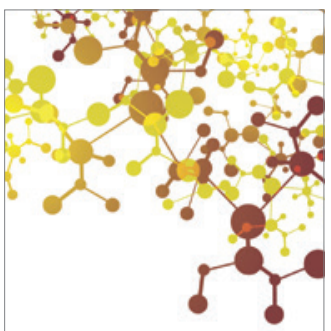

Journal of

Applied Chemistry

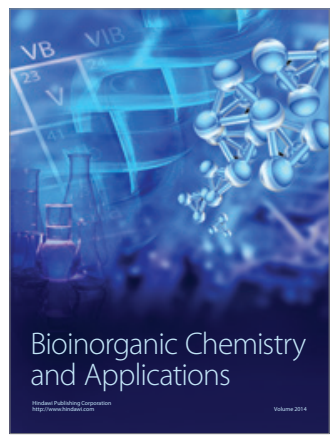

Inorganic Chemistry
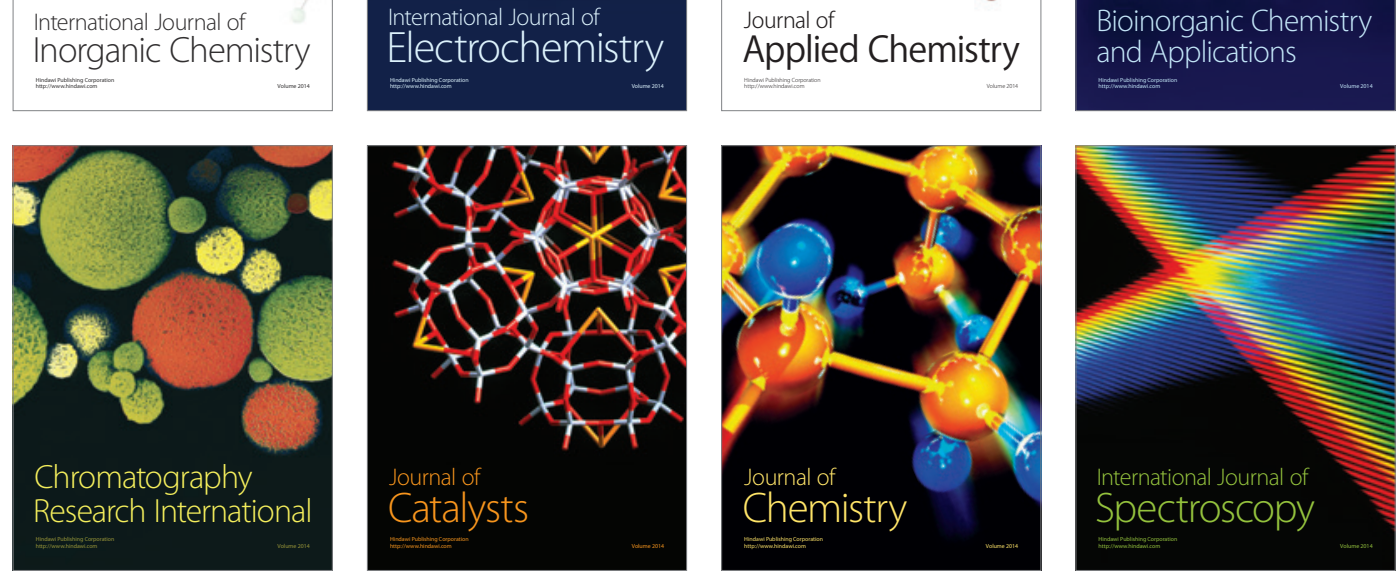